\section{P28 POSTPARTUM PAIN RELIEF MANAGEMENT AND SELF-MEDICATION ON POST-NATAL WARD}

Safia Neetoo, Ofran Almossawi. North Middlesex University Hospital

\subsection{6/archdischild-2015-308634.36}

Introduction During the first few post-operative days after a C-section, adequate pain relief should be provided to the mother to allow speedy recovery and improve mobility to facilitate infant care. The National Institute for Health and Clinical Excellence (NICE) has made recommendations for postpartum women to provide effective pain relief for C-section women.

Self-medication is when patients are responsible for taking their own medications during their hospital stay. Self-medication can be used to improve patients' knowledge about their medicines, to allow patients to be in control of taking their medications during their hospital stay.

Aims To assess whether self-medication offers more effective pain control compared to non self-medicating patients on the post-natal ward at the North Middlesex University Hospital.

To assess the quality of pain management in post-operative $\mathrm{C}$-section women through patient satisfaction questionnaires.

Method The study was carried out on Mondays to Fridays over 1.5 months from Dec to Feb 2012. Thirty non self-medicating women (NSM) and 30 self-medicating women (SM) who had undergone $\mathrm{C}$-sections were enrolled for the study. In the NSM group, nurses administered their medications as per routine practice. In the SM group, women kept their medications at their bedside cabinets and were trained on how to administer them. Patients with substance abuse, social and mental health issues, under the age of 18 years, those who could not administer their own medicines and those with language barriers were excluded from the study.
Data was collected using questionnaire forms; the main outcome measures were global pain scores $(0-10 ; 0=$ no pain, $10=$ worst pain) and patient satisfaction. Nurses also completed a questionnaire on self-medication and pain relief.

Results Baseline age, parity and pain scores were similar in both groups. Mean (SD) global pain score after medication in SM and NSM groups were 4.13 (3.25) and 3.33 (1.77) respectively. The differences in pain scores before and after taking medication in the NSM group were not significant (Mann-U $\mathrm{p}=0.08$ ); but statistically significant in the $\mathrm{SM}$ group, $\mathrm{p}=0.001$. Satisfaction scores in the SM group were significantly higher than in the NSM group. Satisfaction scores over four categories, namely; empowerment over pain control $(\mathrm{p}=0.0006)$, appropriateness of pain control $(p=0.006)$, timing of pain medication (0.006), and overall satisfaction $(p=0.03)$ were all higher in the SM group.

Conclusion Self-medication offers better pain control, education of patients about their medications, improves patient satisfaction and facilitates discharge. Patients are significantly satisfied with self-medicating and are empowered by being in control of their own pain. Self-medication is widely accepted both by patients and nurses; it can therefore be used as an alternative method of postpartum pain control which extends patient choice about their care. 